\title{
Analysis and Implementation of Bus Topology in OPNET Simulator Environment
}

\author{
Rima Shelat ${ }^{1}$ and Megha Bhatt ${ }^{2}$
}

Abstract- A network is a combination of computers and devices for the purpose of sharing data, communication, and resource sharing. In this work, the performance Analysis of Bus topology has been done through the simulation process. The work was initiated with finding the performance of Bus network for the various parameters Delay (Sec), Traffic Sent (bits/Sec), Traffic Received (bits/Sec), throughput (bits/Sec) for the 10 Ethcoax Nodes using the OPNET simulator. The analysis is performed for 1 hour duration and the results were analyzed.

Keywords: OPNET, Bus Topology, Load, Traffic Sent, Traffic Receive, Delay, Nodes.

\section{Introduction}

The Bus topology is one of the fundamental structures of Local Area Network (LAN), which was used in a limited area with a limited number of nodes. In the Earlier days the topology used to be created with a limited number of nodes. Now a day it has changed to more number of nodes to be connected to a LAN Environment. Bus topology is a topology which broadcasts the message among the number of users. In bus topology, each user is connected to an Ethernet Coaxial Cable which is also known as a Backbone. Here the process of data transmission between 10 number of nodes has been simulated and the results are evaluated through the simulation. According to the nature of Bus topology, there may be many chances of network congestion creation as the Bus topology transmits the data among every user. When it finds the $\mathrm{T}$ connection while passing through the backbone the message is broadcasted. There are terminators at the both ends of the topology which defines the end of the topology. Bus topology is not expensive, but to add a node into the topology is easy too. To add a new connection the network needs to be off.

\section{Problem Definition and Scope}

\section{Problem Definition:}

The Bus topology broadcasts the data to every user due to that there may be a chance of a traffic creation. With this work, A bus topology was investigated with 10 number of nodes and their performance of nodes were investigated and Load (bits/Sec) was evaluated for the nodes and results for Traffic Sent (bits/Sec), Traffic Received (bits/Sec), Throughput (bits/Sec) was offered. The simulation duration was selected for 1 hour for optimizing the results of the above parameters.

\footnotetext{
${ }^{1}$ Bholabhai Patel College of Computer Studies Gandhinagar, Gujarat, India

${ }^{2}$ Bholabhai Patel College of Computer Studies Gandhinagar, Gujarat, India
} 


\section{Objectives:}

- Delay (Sec): The end to end delay of frames accepted by all ethernet layers in the network.

- Traffic Sent (bits/Sec): Traffic Sent (in bits/Sec) by the traffic sources across all the nodes.

- Traffic Received (bits/Sec): Traffic received (in bits/Sec) by the traffic sinks across all nodes.

- Throughput (bits/Sec): This statistic represents the average number of bytes successfully received or transmitted by the receiver or the transmitter channel per unit time, in bits per Sec.

- Load (bits/Sec): Average rate of traffic submitted to the specified Ethernet layer by the next- higher level node.

- Performance Analysis of Bus Network

In this work, A bus topology of 10 nodes has been selected for investigation for checking the Ethernet parameters. The selection criteria of bus network are based on the Literature Review done and by the nature of the topology. The parameters which are to be investigated in this work: Delay (Sec), Load (bits/Sec), Traffic Sent (bits/Sec), Traffic Received (bits/Sec), Throughput (bits/Sec).

The figure below shows the topology rapid configuration with 10 number of nodes in ethernet workstations.

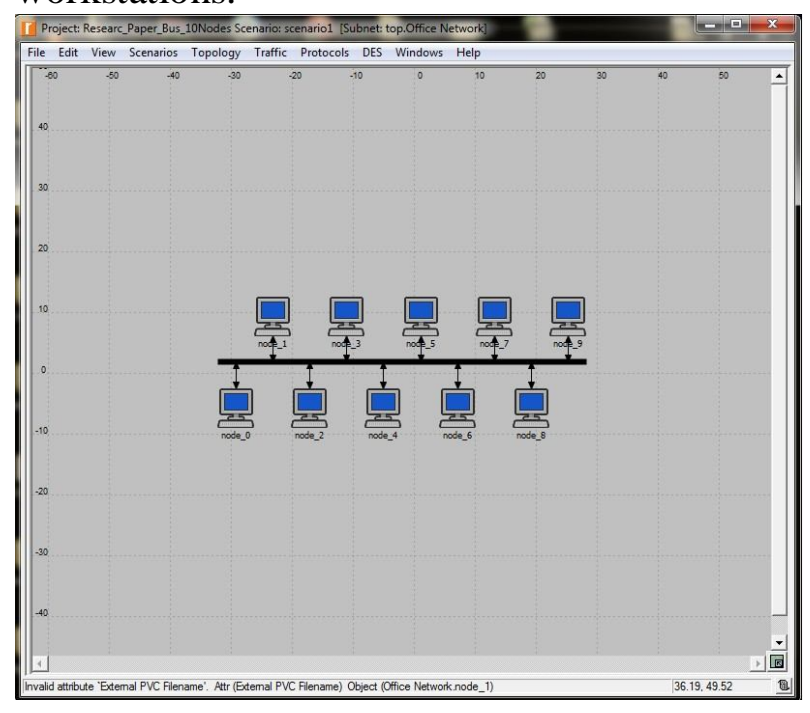

(Fig 1: A View of Bus Topology Design in OPNET)

Parameter selection in OPNET is: - Delay (bits), Load (bits/Sec), Traffic Sent (bits/Sec), Traffic Received (bits/Sec), Throughput (bits/Sec). 


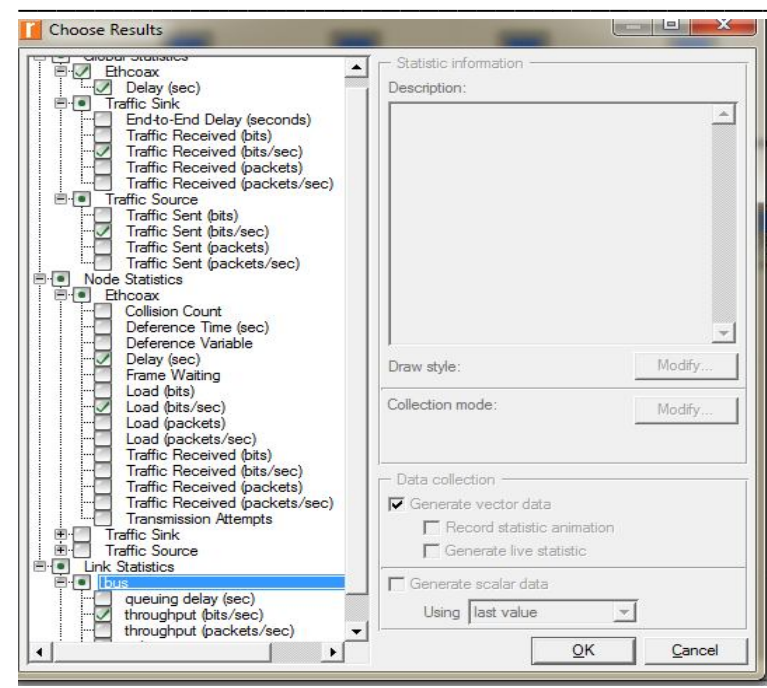

(Fig 2: Parameter Selection in OPNET)

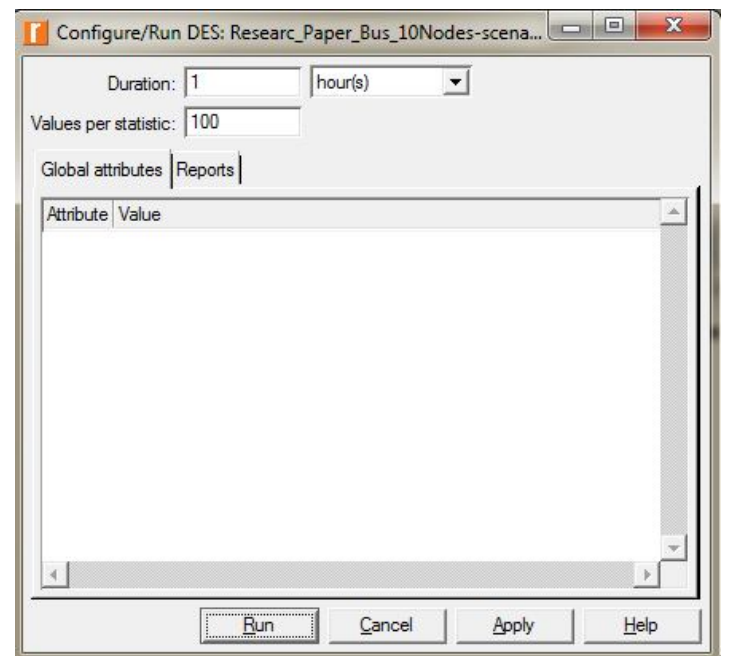

(Fig 3: Configure simulation time in OPNET)

Over laid analysis of Traffic Sent (bits/Sec) and Traffic Received (bits/Sec) for Bus Network.

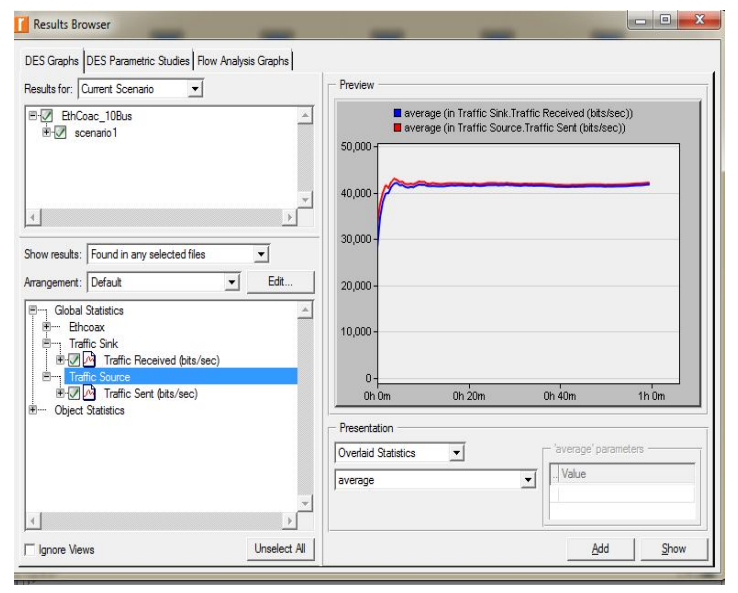


(Fig 4: Overlaid statistics of Bus network for Traffic Sent (bits/Sec), Traffic Received (bits/Sec) in OPNET) Stacked analysis of Delay (bits) in Bus Network.

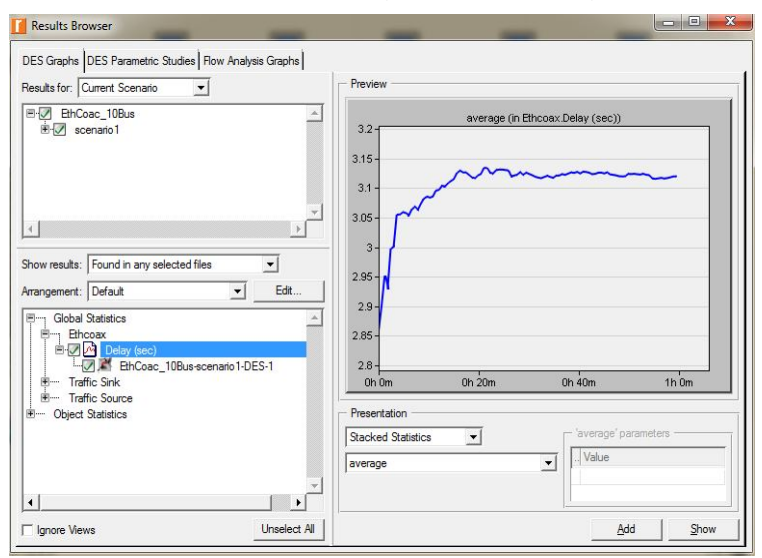

(Fig 5: Stacked Analysis of Bus network for Delay (Sec) in OPNET)

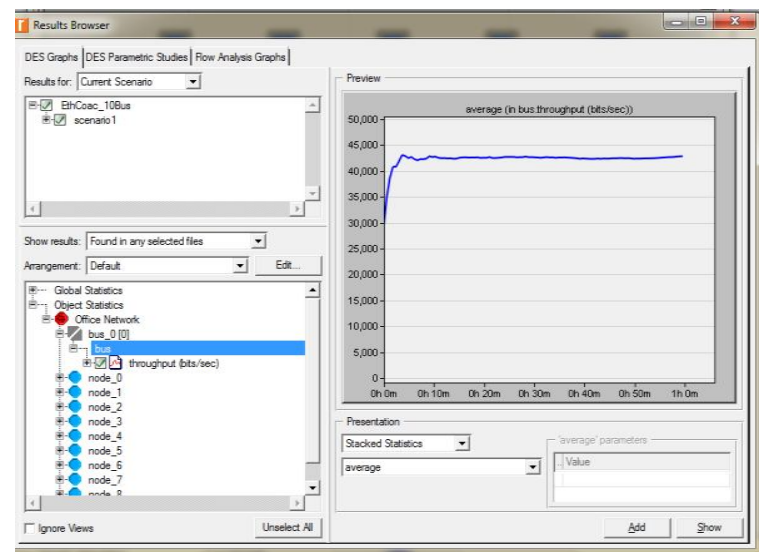

(Fig 6: Overlaid analysis of Throughput (bits/Sec) in Bus Network)

For the extensive analysis of the various node performance the overlaid statistics of Load (bits/Sec) for Node 0 to Node 9.

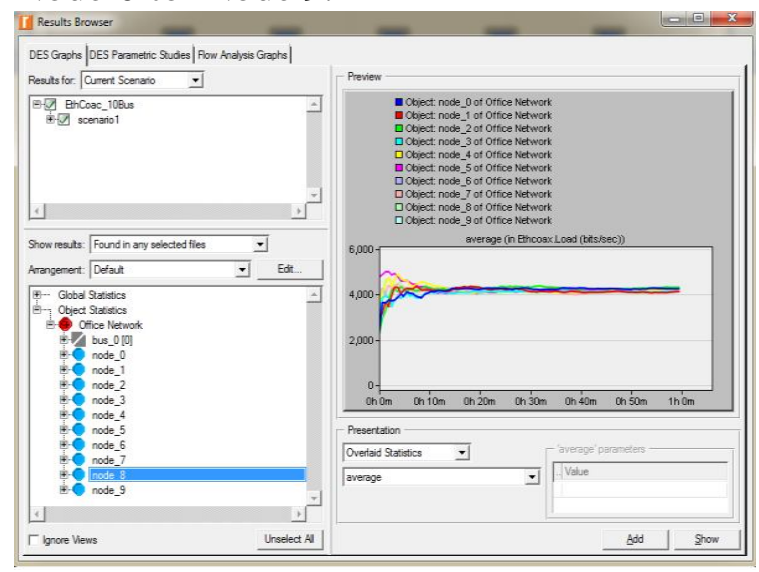

(Fig 7: Overlaid analysis of Load (bits/Sec) for Node 0 to Node 9 in OPNET) 
For the wide analysis of the various node performance the overlaid statistics of Delay (bits) for Node 0 to Node 9.

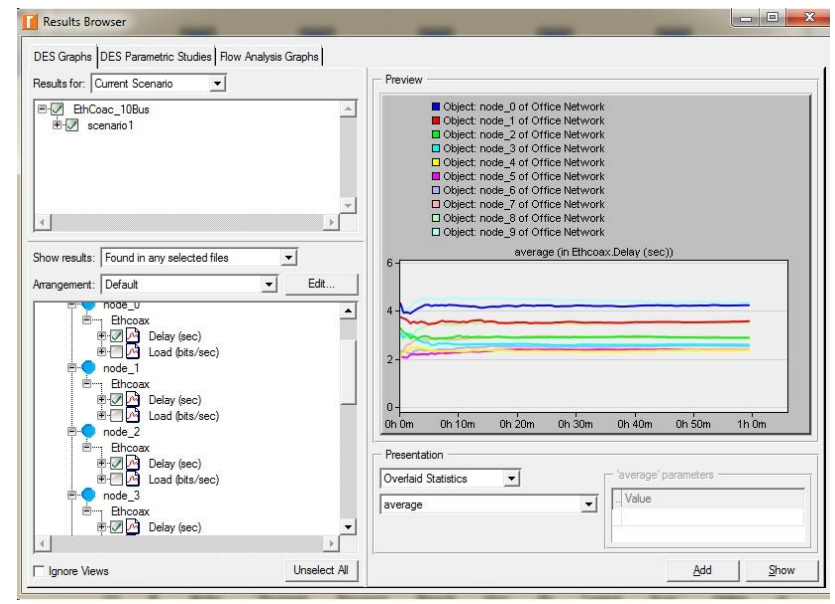

(Fig 8: Overlaid analysis for Delay (Sec) for Node 0 to Node 9) 
In this research work, a Bus topology of 10 nodes has been taken into consideration to evaluate its ethernet parameters. The parameters evaluated are:

Delay (Sec), Load (bits/Sec), Traffic Sent (bits/Sec), Traffic Received (bits/Sec), Throughput (bits/Sec) for Ethernet network have been evaluated for node 0, 1, 2, 3, 4, 5, 6, 7, 8, 9 .

For extended analysis, Overlaid statistics for Load (bits/Sec), Delay (bits/Sec) for individual nodes has been investigated.

In this work, the Bus topology was investigated with a coaxial cable link for a different number of nodes. It was found that wired network performs well within a limited number of range with a limited number of connections and the impact was found in OPNET simulator. As the number of nodes increases the performance of the network degrades.

\section{Conclusions and Future Scope}

The various factors which affect the performance of the wired network has been evaluated using the OPNET simulator. The results prove that the configuration of a network in OPNET simulator is much easier than the real time implementation. The main conclusion is As the number of nodes increases the performance of the network is degraded. Though there is a minor difference in the Load (bits/Sec) for every node, which is shown in fig 7. And there not much difference in End to End Delay (Sec) for every node, when small numbers of nodes are selected for a network creation.

This paper mainly focuses on the performance analysis of the Wired Bus Network. In future the same parameters can be utilized to measure the performance of other LAN Topology such as Star, Ring, Mesh and for different number of nodes in OPNET simulator.

\section{References}

[1] Harmeet Singh, Sukhjeet Singh, Rahul Malhotra, "Modeling evaluation and analysis of Ring topology for computer applications using simulation,” in IJCSMC, Jan 2013, pp. 1-10..

[2] "A Survey of NetworkSimulation Tools: Current Status and Future Developments," Online at http://www.cs.wustl.edu/ jain/cse567-08/ftp/simtools.pdf, 2016.

[3] Othman AL-Rusaini and Adnan Afridi, "Studying the performance of a local area network through a statistical result of a simulation program,"'in ISOR journal of computer engineering, MayJune 2016, pp. 42-57..

[4] A.A. Atayero, A.S Alatishe, J.O Iruemi, "Modeling and simulation of a University LAN in OPNET modeler Environment, Department of Electrical and Information Engineering", September 2016. 